\title{
The Debye-Waller factor of liquid silica: Theory and simulation
}

\author{
Francesco Sciortino* and Walter $\mathrm{Kob}^{\dagger}$ \\ * Dipartimento di Fisica and Istituto Nazionale per la Fisica della Materia, Universitá di Roma La Sapienza, P.le Aldo Moro \\ 2, I-00185 Roma, Italy. \\ ${ }^{\dagger}$ Institute of Physics, Johannes Gutenberg-University, Staudinger Weg 7, D-55099 Mainz, Germany
}

(24. June, 2000)

We show that the prediction of mode-coupling theory for a model of a network-forming strong glass-former correctly describes the wave-vector dependence of the Debye-Waller factor. To obtain a good description it is important to take into account the triplet correlation function $\mathbf{c}_{3}$, which we evaluate from a computer simulation. Our results support the possibility that this theory is able to accurately describe the nonergodicity parameters of simple as well as of network-forming liquids.

PACS numbers: 61.20.Lc, 61.20.Ja, 64.70.Pf, 51.10.+y

The quantitative description of the glassy dynamics in liquids is one important goal of modern research in condensed matter. Work in the last decade has provided evidence [1] that the so-called mode-coupling theory (MCT) 20 is able to describe the slow dynamics of fragile liquids in the weakly supercooled state. Detailed theoretical predictions for model systems - including hard sphere systems, simple binary liquids, and molecular liquids - have been found to be in remarkable agreement with experimental measurements as well as with simulation results [3 6]. E.g. the full $q$-dependence of the Lamb-Mössbauer and of the Debye-Waller factors is predicted well by the theory.

Recently, it has been shown that also intermediate and strong glass-formers, such as glycerol or silica $\left(\mathrm{SiO}_{2}\right)$ [8,9], show features that are in qualitative agreement with the predictions of MCT. However, in the case of silica, a detailed comparison between theory and numerical data has questioned the ability of MCT to describe correctly the Debye-Waller factors of this important network-forming material 10,11]. In this Letter we show that the disagreement between MD-results and theoretical predictions - for the case of silica - were not due to failure of MCT to describe caging in this network forming liquid but to a further approximation which is assumed for the sake of simplicity in the commonly-used MCT equations. We show that, once this approximation is avoided, MCT is able to describe accurately the cages in silica, opening the way for a full description of dynamics in network forming liquids above the critical temperature of MCT.

To start we briefly review the MCT equations. The central quantity is the coherent intermediate scattering function $\mathbf{F}(\mathbf{q}, t)$, which for binary system can be written as a $2 \times 2$ matrix with entries $F_{i j}(\mathbf{q}, t)=$ $\left\langle\delta \rho_{i}(\mathbf{q}, 0) \delta \rho_{j}^{\star}(\mathbf{q}, t)\right\rangle$. Here $\rho_{i}(\mathbf{q}, t)$ is the density fluctuations for wave-vector $\mathbf{q}$, and $i$ is the label for the species.

The equation of motion for $\mathbf{F}(\mathbf{q}, t)$ is given by

$\ddot{\mathbf{F}}(\mathbf{q}, t)+\boldsymbol{\Omega}^{2}(q) \mathbf{F}(\mathbf{q}, t)+\int_{0}^{t} d \tau \mathbf{M}(\mathbf{q}, t-\tau) \dot{\mathbf{F}}(\mathbf{q}, \tau)=0$,

where the frequency matrix is given by $\left[\boldsymbol{\Omega}^{2}(q)\right]_{i j}=$ $q^{2} k_{B} T\left(x_{i} / m_{i}\right) \sum_{k} \delta_{i k}\left[\mathbf{S}^{-1}(q)\right]_{k j}$. Here $q=|\mathbf{q}|, x_{i}$ is the concentration of species $i, m_{i}$ is their mass, and $\mathbf{S}$ is the partial structure factor matrix. Within the framework of MCT, the memory function $\mathbf{M}$ is given by $M_{i j}=x_{i} k_{B} T N_{i j} / m_{i}$, where the matrix $N_{i j}$ is a quadratic form in $F_{i j}(\mathbf{q}, t)$ :

$$
\begin{aligned}
N_{i j}(\mathbf{q}, t)= & \frac{1}{2 n x_{i} x_{j}} \int \frac{d \mathbf{k}}{(2 \pi)^{3}} \sum_{\alpha \beta} \sum_{\alpha^{\prime} \beta^{\prime}} V_{i \alpha \beta}(\mathbf{q}, \mathbf{k}) \times \\
& V_{j \alpha^{\prime} \beta^{\prime}}(\mathbf{q}, \mathbf{q}-\mathbf{k}) F_{\alpha \alpha^{\prime}}(\mathbf{k}, t) F_{\beta \beta^{\prime}}(\mathbf{q}-\mathbf{k}, t) .
\end{aligned}
$$

Here $n$ is the particle density, and the vertices $V_{i \alpha \beta}(\mathbf{q}, \mathbf{k})$ are given by

$$
\begin{aligned}
V_{i \alpha \beta}(\mathbf{q}, \mathbf{k})=\frac{\mathbf{q} \cdot \mathbf{k}}{q} \delta_{i \beta} c_{2}^{i \alpha}(\mathbf{k})+ & \frac{\mathbf{q} \cdot(\mathbf{q}-\mathbf{k})}{q} \delta_{i \alpha} c_{2}^{i \beta}(\mathbf{q}-\mathbf{k}) \\
& +n q c_{3}^{i \alpha \beta}(\mathbf{q}, \mathbf{q}-\mathbf{k}) \cdot x_{i} .
\end{aligned}
$$

The function $c_{2}^{i j}(\mathbf{q})=\delta_{i j} / x_{i}-\left[\mathbf{S}^{-1}(\mathbf{q})\right]_{i j}$ is the direct correlation function, and $c_{3}^{i \alpha \beta}(\mathbf{q}, \mathbf{k})$ is the triple correlation function, which is related to the triple density fluctuations via

$$
\begin{aligned}
& \left\langle\delta \rho_{\alpha}(\mathbf{q}) \delta \rho_{\beta}(\mathbf{k}) \delta \rho_{\gamma}^{\star}(\mathbf{q}+\mathbf{k})\right\rangle=N \sum_{\epsilon \sigma \eta} S_{\alpha \epsilon}(q) S_{\beta \sigma}(k) \\
& \times S_{\gamma \eta}(|\mathbf{q}+\mathbf{k}|)\left(\delta_{\epsilon \sigma} \delta_{\epsilon \eta} \delta_{\eta \sigma} / x_{\epsilon}^{2}+n^{2} c_{3}^{\epsilon \sigma \eta}(\mathbf{q}, \mathbf{k})\right) .
\end{aligned}
$$

The only input required by $\mathrm{MCT}$ are the static quantities $\mathbf{S}, \mathbf{c}_{3}$, and $n$. Temperature enters the equations only through the explicit $T$-dependence of $\boldsymbol{\Omega}^{2}(q)$ and the implicit $T$-dependence of the static quantities. The solution of this type of equation shows at low $T$ a two-step relaxation dynamics, if $T$ is above $T_{c}$, the so-called critical temperature of MCT [2]. Below $T_{c}$ the time correlation functions do not decay to zero anymore, thus signaling that the system is not longer ergodic. Hence the height of the plateau in a time correlation function is usually called the non-ergodicity parameter (NEP) since it measures that fraction of the correlation that does not decay 
to zero even at long times. The physical relevance of the NEP can be inferred from the fact that it can be directly measured in light and neutron scattering experiments. For the case of $F(\mathbf{q}, t)$ the $\mathrm{NEP}$ is $\mathbf{F}_{c}(q)$, the Debye-Waller factor for wave-vector $q$.

In one of the first attempts to solve the MCT equations for soft spheres, the possibility of setting in Eq. (3) $\mathbf{c}_{3}=0$ was considered [3]. This approximation corresponds to a factorization of the triple density correlation in $q$-space in product of the three pair density correlation. It was found that for this simple liquid, this approximation does not significantly affect the MCT predictions. All further works have build on this information. In this Letter we check the validity of this approximation for network-forming liquids. We use molecular dynamics (MD) simulations to determine the triple correlation function for silica [12] and use these functions to solve the full MCT equations. This calculation allows us to determine for the first time whether or not MCT is able to give a quantitative description of the cages of strong glass-formers. For completeness, we perform the same study for a well studied binary mixture of Lennard-Jones particles (BMLJ) 16.

In the past, the dynamics of both systems has been carefully analyzed and it has been shown that the relaxation dynamics shows the qualitative features predicted by the MCT $99,11,17,18]$. For the case of the BMLJ also a quantitative comparison has been made in that $\mathbf{F}_{c}(q)$ has been calculated theoretically (assuming $\mathbf{c}_{3}=0$ ) and compared with simulation data. This comparison showed that very good agreement between theory and simulation is obtained, even if $\mathbf{c}_{3}=0$ is assumed, in agreement with the conclusions of Barrat and Latz [3]. But for the case of $\mathrm{SiO}_{2}$ a similar comparison showed that the MCT prediction for $\mathbf{F}_{c}(q)$ does not describe properly the confining cage if $\mathbf{c}_{3}=0$ 10,11.

In order to investigate the influence of $\mathbf{c}_{3}$ it is necessary to determine $c_{3}^{\epsilon \sigma \eta}(\mathbf{q}, \mathbf{k})$ with high precision. For this we calculated $\left\langle\delta \rho_{\alpha}(\mathbf{k}) \delta \rho_{\beta}(\mathbf{p}) \delta \rho_{\gamma}^{\star}(\mathbf{q})\right\rangle$ with $\mathbf{q}=\mathbf{k}+\mathbf{p}$ for all triplets of values $|\mathbf{k}|,|\mathbf{p}|,|\mathbf{q}|$, with $|\mathbf{p}| \geq|\mathbf{k}|$ and $|\mathbf{p}-\mathbf{k}|<|\mathbf{q}|<|\mathbf{p}+\mathbf{k}|$, and then used Eq. (4) to determine $\mathbf{c}_{3}$. We have selected a mesh size $\Delta$, where $\Delta$ was $0.154 \AA^{-1}$ and 0.334 for the case of $\mathrm{SiO}_{2}$ and BMLJ, respectively. Only wavevectors with modulus less than $100 \Delta$ have been considered. For each triplet of value for $|\mathbf{k}|,|\mathbf{p}|,|\mathbf{q}|$ we randomly selected up to $10^{2}$ combinations of wave-vectors $\mathbf{k}, \mathbf{p}, \mathbf{q}$, calculated for these the density fluctuations and then the triple density correlation function. For BMLJ this was done for 12309 completely independent configurations, corresponding to 100 million time steps in the simulation. For $\mathrm{SiO}_{2}$ we analyzed 8577 configurations (20 million time steps). Note that this very large number of independent configurations was needed in order to determine $\mathbf{c}_{3}$ with sufficient high accuracy to make sure that the final results for the NEP do not depend anymore on the noise in $\mathbf{c}_{3}$.
We mention that using only 1000 independent configurations was, e.g., not sufficient to guarantee this. Due to the large effort needed to generate the configurations and to analyze them, we determined $\mathbf{c}_{3}$ only for one temperature, namely $T=1.0$ (BMLJ) and $T=4000 \mathrm{~K} \mathrm{SiO}_{2}$. Thus in the calculation of the MCT vertices in Eq. (3) we assumed that the direct correlation functions depend on temperature but that the $T$-dependence of $\mathbf{c}_{3}$ can be neglected.

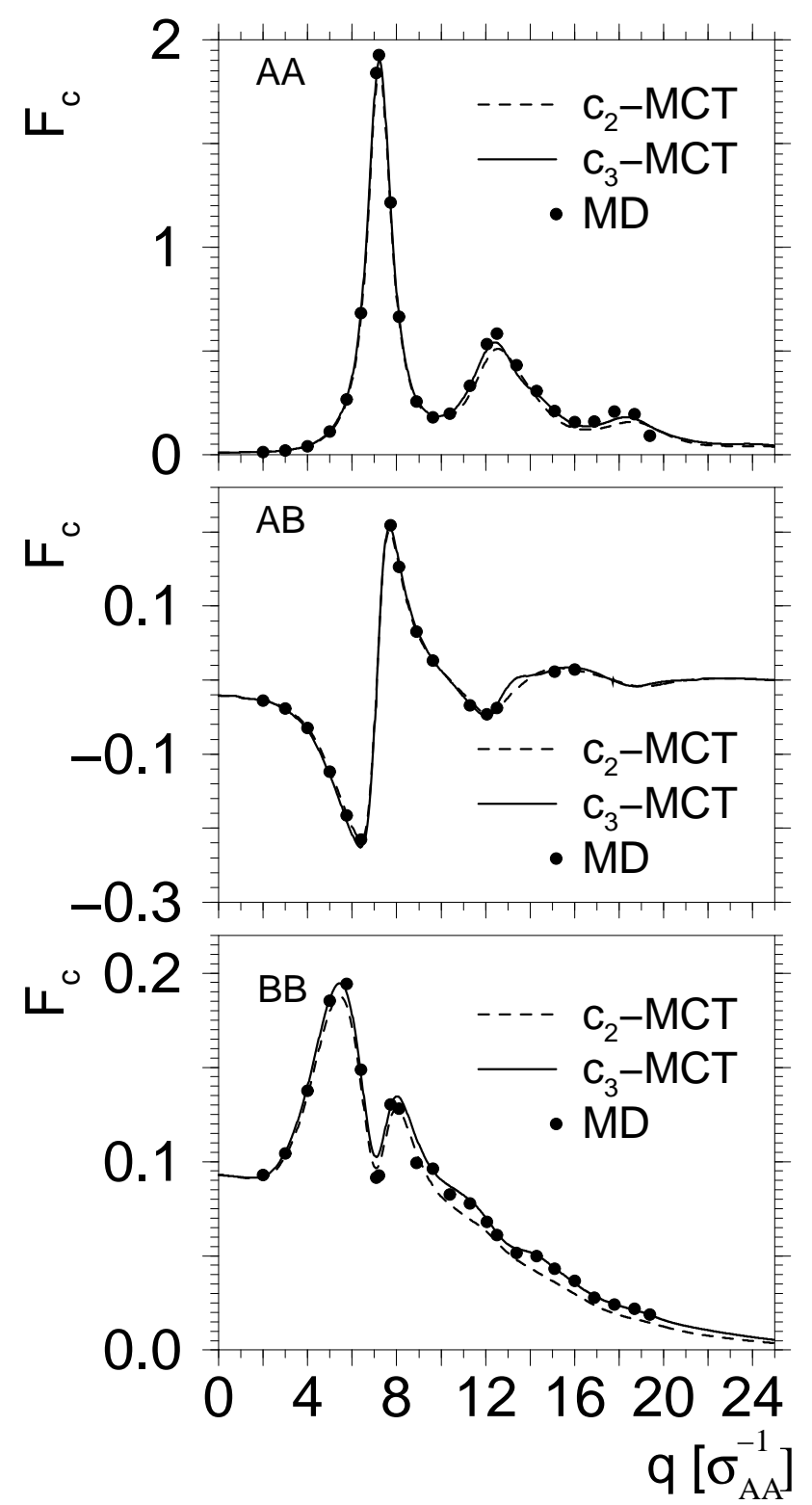

FIG. 1. Wave-vector dependence for the non-ergodicity parameters for the BMLJ system. The solid and dashed curves are the theoretical prediction with and without the inclusion of the $\mathbf{c}_{3}$ terms in Eq. (2). The circles are the MD results from 18 . 


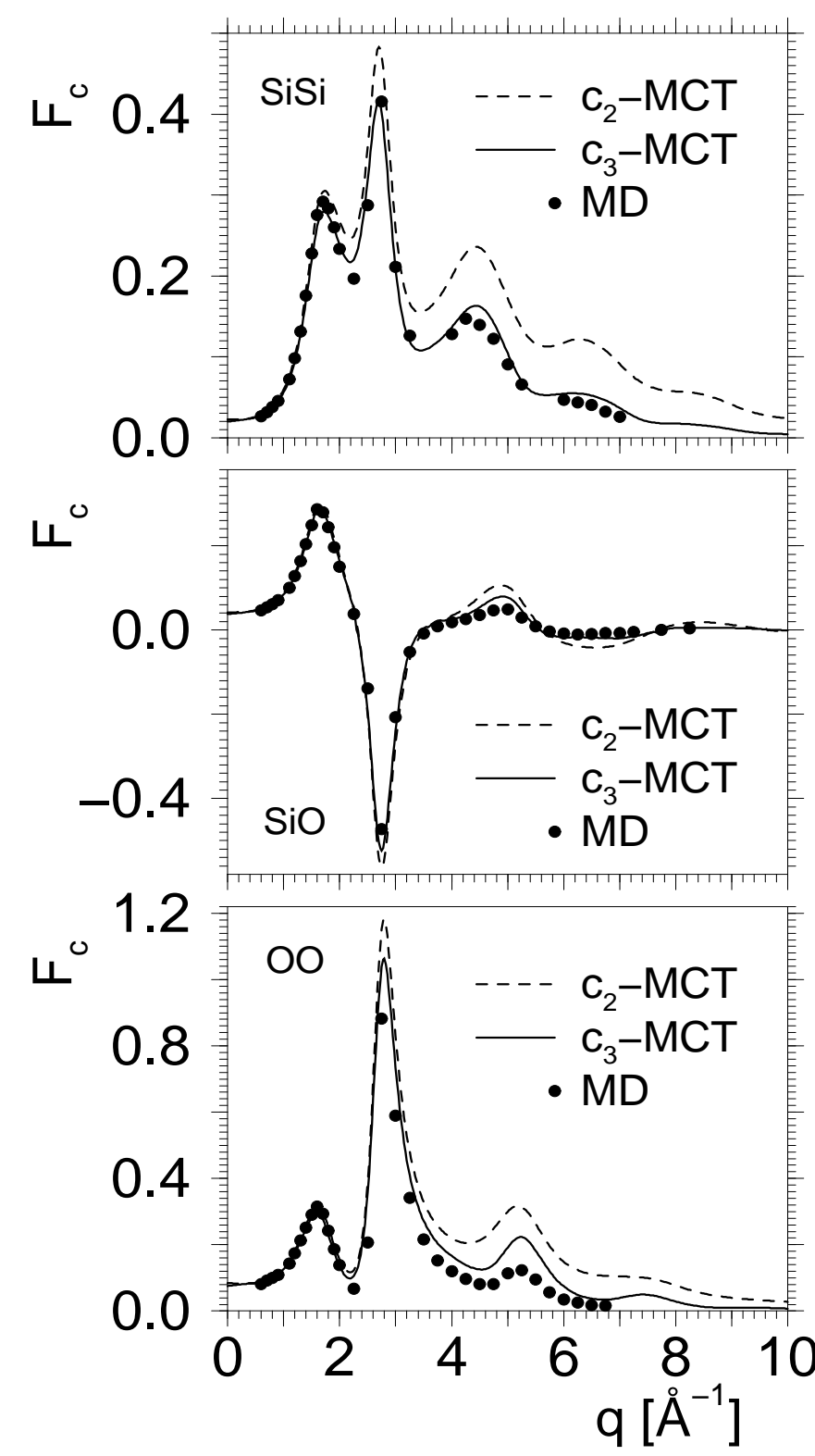

FIG. 2. The same quantities as in Fig. 1, but for silica. The MD data is from Ref. 10.

From the equations of motion, Eqs. (11)-(3), we determined $T_{c}$ and $\mathbf{F}_{c}(q)$, using the iteration procedure from Ref. [3]. The results obtained are presented in Figs. 1] and 2 were we show the three components of the NEP for the case of the BMLJ and silica, respectively. In each panel three curves are shown: the data from the simulation (filled circles) [18], the theoretical prediction if $\mathbf{c}_{3}=0$ (curves labeled with " $c_{2}$ "), and the theoretical prediction if $\mathbf{c}_{3}$ is taken into account (curves labeled with " $c_{3}$ ") [19]. From Fig. 1 we see that the curves for $c_{2}$ and $c_{3}$ are very close together, from which we conclude that in the case of the BMLJ the inclusion of $\mathbf{c}_{3}$ changes the prediction of the theory only weakly. The main difference between the two curves is in the $B B$ correlation, since the $c_{3}$-curve describes the simulation data even better than the $c_{2}$-curve. Also the theoretical prediction for $T_{c}$ is es- sentially independent of whether or not $\mathbf{c}_{3}$ is neglected, in that $T_{c}$ decreases from $T_{c}=0.922$ to $T_{c}=0.910$ if $\mathbf{c}_{3}$ is taken into account. These results are in agreement with the findings of Ref. [3].

From Fig. 2 we recognize that for the case of $\mathrm{SiO}_{2}$ the influence of $\mathbf{c}_{3}$ on the NEP is much stronger than for the BMLJ system. For this network-forming liquid the inclusion of $\mathbf{c}_{3}$ into the vertices leads to predicted $\mathbf{F}_{c}(q)$ which are in substantially better agreement with the ones from the simulation than the ones from the $c_{2}$-theory. The improvement is particularly noticeable for $q$ above the location of the first peak, a length scale that corresponds to the distance between two neighboring tetrahedra. E.g. in the case of the $\mathrm{Si}$-Si correlation the relative difference between the theoretical curve and the MD data is decreased by a factor around five. The remaining difference is now only on the order of the error of the MD data. Note that $\mathbf{F}_{c}(q)$ provides information on the cage in which a particles is confined and which is formed by its neighboring particles. The fact that in the vicinity of the first maximum the two theoretical curves as well as the MD data are very close together shows that already the $c_{2}$-theory is able to capture the structure of the cage on this length scale. For larger wave-vectors the $c_{2}$-curve is too high, which means that the size of the cage is underestimated. Only if the terms due to $\mathbf{c}_{3}$ are taken into account, a reliable description for the cage is obtained.

For the Si-Si correlation the mentioned decrease of the NEP is more pronounced than for the two other correlation functions, which is reasonable since it is the $\mathrm{Si}$-atoms that sit in the center of the tetrahedra, i.e. that make up the network structure, and hence it can be expected that the inclusion of the $\mathbf{c}_{3}$-terms is important. Nevertheless, from the figure we see that the inclusion of these terms lead also to a significant improvement of the theoretical prediction for the NEPs for Si-O and O-O if $q$ is larger than the location of the first peak. E.g. for the case of $\mathrm{O}-\mathrm{O}$ the relative error of the $c_{3}$-curve for $q \geq 2.0 \AA$ is only half as large as the one for the $c_{2}$-curve. Thus we conclude that the $c_{2}$-MCT is able to give a good description of the cage for the oxygens only on the length scale corresponding to the first maximum of $F(q, 0)$.

The strong influence of the $\mathbf{c}_{3}$-terms on $\mathbf{F}_{c}(q)$ is also found in the value of $T_{c}$. If one sets $\mathbf{c}_{3}=0$ one finds $T_{c}=$ $3962 \mathrm{~K}$, whereas the $c_{3}$-theory predicts $T_{c}=4676 \mathrm{~K}$. Thus, whereas for the BMLJ system we found only a change of the order of $1 \%$, we now find a change around $18 \%$. We mention, however, that MCT usually overestimates the value of $T_{c}$, and indeed we have $T_{c}^{M D}=0.435$ and $T_{c}^{M D}=3330 \mathrm{~K}$ for BMLJ and $\mathrm{SiO}_{2}$, respectively 17. 9 . 

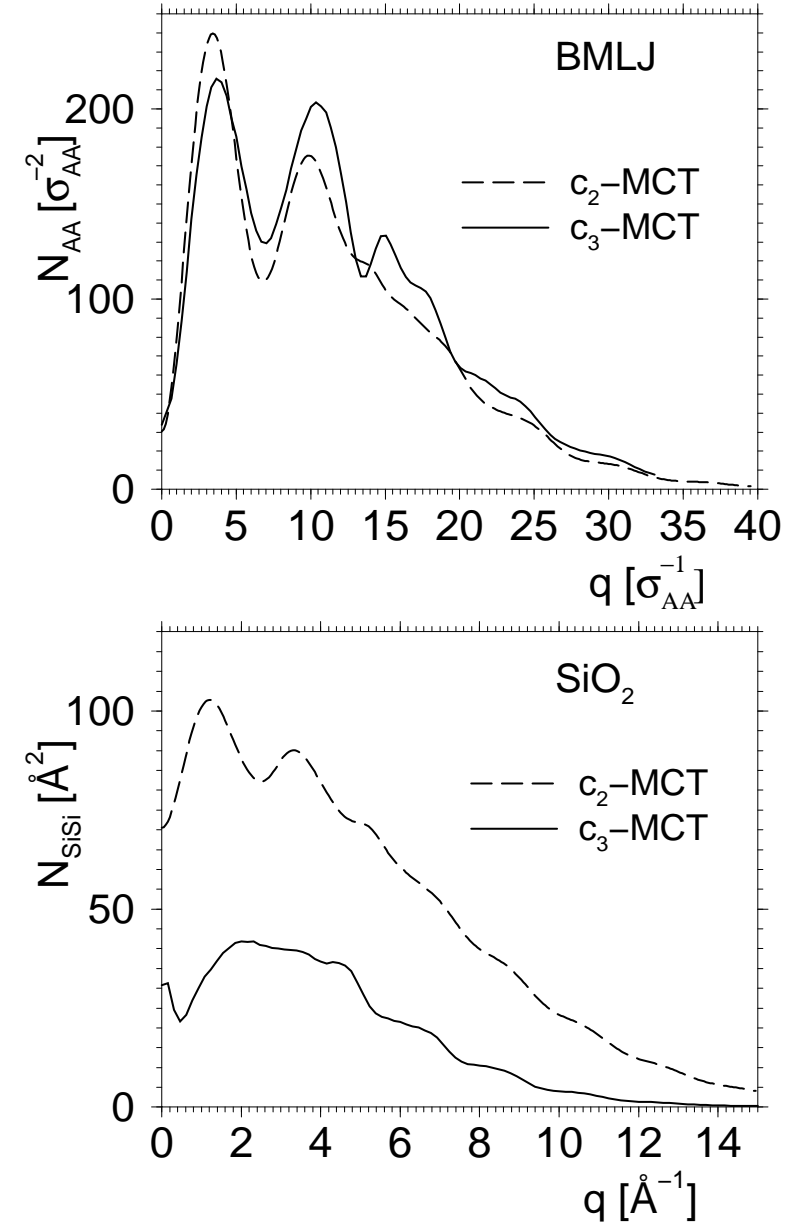

FIG. 3. Wave-vector dependence of the A-A and Si-Si component of the memory function (upper and lower panel, respectively). The dashed and solid curves are for the version of MCT in which the $\mathbf{c}_{3}$-terms are neglected and taken into account, respectively.

Finally we show in Fig. 3 the $q$-dependence of $N_{\mathrm{AA}}$ and $N_{\mathrm{SiSi}}$ from Eq. (2) for the corresponding $T_{c}$. From this figure we see that for the BMLJ case the $c_{2}$-memory function is very similar to the one in which $\mathbf{c}_{3}$ is taken into account and thus it is not surprising that also the NEP and the $T_{c}$ are not changing. This is in stark contrast to the $\mathrm{SiO}_{2}$ system, for which the memory functions from the $c_{2}$ - and $c_{3}$-theory are very different. Note that in a binary mixture each component of the memory function has contributions from 16 different terms (see Eq. (2)). We found that in the case of $\mathrm{SiO}_{2}$ the inclusion of the $\mathbf{c}_{3}$-terms leads to a significant change of the contribution involving the off-diagonal terms. In the $c_{2}$-case these contributions are negative, thus they drive the transition to lower temperatures. If the $\mathbf{c}_{3}$-terms are taken into account, these contributions become less negative and hence the transition takes place already at higher temperatures.

In summary, the present calculation shows that MCT is able to give an accurate quantitative description of the
NEP for a strong glass-former. This opens the way for a detailed description of the dynamics in the MCT region for this class of materials. Hence our results support the possibility that $\mathrm{MCT}$ is able to make quantitative predictions for all types of glass-formers.

Acknowledgment: We thanks L. Fabbian and M. Nauroth for contributing to the early development of this research, and J. Horbach for providing the $\mathrm{SiO}_{2}$ NEPs. Part of this work was supported by the DFG through SFB 262. F.S. acknowledges support from INFM-PRAHOP and MURST-PRIN98. W. K. thanks the Universitá La Sapienza for a visiting professorship during which part of this work was carried out.

[1] See, e.g., Proceedings of Third International Discussion Meeting, J. Non-Cryst. Solids 235-237, (1998).

[2] W. Götze, J. Phys.: Condens. Matter 10, A1 (1999).

[3] J.-L. Barrat and A. Latz, J. Phys.: Condens. Matter 2, 4289 (1990).

[4] W. van Megen and S. M. Underwood, Phys. Rev. E 49, 4206 (1994).

[5] M. Nauroth and W. Kob, Phys. Rev. E 55, 657 (1997).

[6] L. Fabbian, A. Latz, R. Schilling, F. Sciortino, P. Tartaglia and C. Theis Phys. Rev. E. 605768 (1999); C. Theis, F. Sciortino, A. Latz, R. Schilling, and P. Tartaglia, Phys. Rev. E (in press).

[7] A. Winkler, A. Latz, R. Schilling, and C. Theis, preprint cond-mat/0007276.

[8] T. Franosch, W. Götze, M. R. Mayr, and A. P. Singh, Phys. Rev. E 55, 3183 (1997).

[9] J. Horbach and W. Kob, Phys. Rev. B 60, 3169 (1999).

[10] J. Horbach, PhD thesis (University of Mainz, 1998).

[11] M. Nauroth, PhD thesis (Technical University Munich, 1999).

[12] The potential used to describe silica is the one proposed by van Beest et al. 13 and which has the functional form $V_{\alpha \beta}=q_{\alpha} q_{\beta} / r+A_{\alpha} q_{\beta} \exp \left(B_{\alpha} q_{\beta} r\right)-C_{\alpha} q_{\beta} / r^{6}$, with $\alpha, \beta \in$ $\{\mathrm{Si}, \mathrm{O}\}$. The values of the parameters $q_{\alpha}, A_{\alpha \beta}, B_{\alpha \beta}$, and $C_{\alpha \beta}$ can be found in Ref. [13. Despite its simplicity, this potential has been shown to give a reliable description of the static and dynamical quantities of real $\mathrm{SiO}_{2}, 9,14,15$. In particular it is able to reproduce its open network structure. In the present simulation we used 600 ions in a box of $(20.4 \AA)^{3}$.

[13] B. W. H. van Beest, G. J. Kramer, and R. A. van Santen, Phys. Rev. Lett. 64, 1955 (1990).

[14] K. Vollmayr, W. Kob, and K. Binder, Phys. Rev. B 54, 15808 (1996).

[15] P. Jund and R. Jullien, Phys. Rev. B 59, 13707 (1999).

[16] The BMLJ system is composed of 1000 particles, $80 \% \mathrm{~A}$ and $20 \% \mathrm{~B}$ particles, with the same mass $m$, which interact via a Lennard-Jones potential of the form $V_{\alpha \beta}(r)=$ $4 \epsilon_{\alpha \beta}\left[\left(\sigma_{\alpha \beta} / r\right)^{12}-\left(\sigma_{\alpha \beta} / r\right)^{6}\right]$, with $\alpha, \beta \in\{\mathrm{A}, \mathrm{B}\}$ and cutoff radii $r_{\alpha \beta}^{\mathrm{c}}=2.5 \sigma_{\alpha \beta}$. The parameters are $\epsilon_{\mathrm{AA}}=1.0$, 
$\sigma_{\mathrm{AA}}=1.0, \epsilon_{\mathrm{AB}}=1.5, \sigma_{\mathrm{AB}}=0.8, \epsilon_{\mathrm{BB}}=0.5$, and $\sigma_{\mathrm{BB}}=0.88$. We use $n=1.2$, with $\sigma_{\mathrm{AA}}$ as the unit of length.

[17] W. Kob and H. C. Andersen, Phys. Rev. E 51, 4626 (1995); Phys. Rev. E 52, 4134 (1995).

[18] T. Gleim, W. Kob, and K. Binder, Phys. Rev. Lett. 81, 4404 (1998).

[19] Note that $\mathbf{F}_{c}(q)$ are not normalized by the static structure factor, since this would give rise to singularities if $S_{\alpha \beta}(q)=0$. To account for the $T$-dependence of $\mathbf{F}(q, 0)$, we first divide $\mathbf{F}_{c}(q)$ by $\mathbf{F}(q, 0)$ at the corresponding $T_{c}$ and then multiply this function by $\mathbf{F}(q, 0)$ of the $c_{2}$-curve. 